\title{
Dynamic and Electrical Analysis of MEMS Capacitor with Accelerated Motion Effects
}

\author{
Kohei Kawano Shafrida Shahrani Takashi Mori Michiko Kuroda \\ Tokyo University of Technology \\ Hachioji, Tokyo, 198-0982, Japan \\ kuroda@,cc.teu.ac.jp \\ Manos M. Tentzeris \\ Georgia Institute of Technology \\ Atlanta, GA,30332-250, USA
}

\begin{abstract}
MEMS technology is growing rapidly in RF field, because of the advantage over p-i-n diode or FET switches. An accurate knowledge of the electromagnetic field evolution around a moving or rotating body is very important for the design, optimization and realization of new optical devices or microwave devices, such as the RF-MEMS structures. We propose the numerical technique based on the finite-difference time-domain method with an adaptive implementation of grid generation. This simulation method is applied to the analysis of a two-dimensional MEMS variable capacitor with accelerated motions. The acceleration of the MEMS capacitor is derived under the equilibrium between the spring force and electrical force. Using this acceleration, the numerical results that express the relationship between the acceleration of the plates and the spring constant and the mass of the plates are shown.
\end{abstract}

Keywords: RF MEMS, body fitted grid generation method with moving boundaries, FD-TD method, acceleration, mechanical resonant frequency

\section{Introduction}

As RF MEMS has many application areas[1],[2], for the modeling and optimization of microwave devices, an accurate knowledge of the electromagnetic evolution around a moving or rotating body is required. But due to the limitations of the conventional numerical technique for the time changing boundaries, it is tedious to solve these problems numerically in electromagnetic field.

We propose the numerical technique based on the finite-difference time-domain method with an adaptive implementation of grid generation [3]. In this paper, this simulation method[4] is applied to the analysis of a two-dimensional MEMS variable capacitor with accelerated motion effect. The acceleration of the plates is derived from the equilibrium between the spring force and electrical force. Using this acceleration, the relation between the mass, the spring constant and the oscillation of the plates are shown. This acceleration is useful to obtain the switching time.

\section{Mechanical and Electrical Equations}


The dynamic behavior of the MEMS structure is shown in Fig.1, and the top plate is suspended by a spring. Under the combined effect of mechanical and electrical force, the top plate moves until the equilibrium between the electrostatic and mechanical forces is reached. $F_{m}$ means spring force and $F_{e}$ means electrostatic force and these forces are expressed in the following equations, respectively[1].

$$
\begin{aligned}
& F_{m}=m x^{\prime \prime}+b x^{\prime}+k x \\
& F_{e}=\frac{1}{2} \frac{\partial C}{\partial x} V^{2}
\end{aligned}
$$

, where $m$ is the mass of the plate and $b$ is the mechanical resistance and $k$ is the spring constant, $V$ is the bias voltage. From the equilibrium between the spring force and the electrostatic force, the following equation is derived.

$$
m x^{\prime \prime}=\frac{1}{2} \frac{\partial C}{\partial x} V^{2}-b x^{\prime}-k x
$$

And from eq.(3), the acceleration $x$ ” is obtained. The geometry to be considered here is shown in Fig2. Under the combined effect of mechanical and electrical force, the two fingers are assumed to move with different velocities for $x$-direction. For the two-dimensional TM-propagation case, as shown in Fig.2, there are only $E x, E y, H z$ nonzero components with a time variation. In Fig.2, the configurations of the physical and of the computational regions are shown.

Under the combined effect of mechanical and electrical force, the plates are assumed to move for $x$ direction with velocities $v$ and $u$, and the acceleration $a_{v}, a_{u}$ respectively. Using a coordinates' transformation technique, the time-changing physical region $(x, y, t)$ can evolve to a time-invariant computational domain. For the geometry of Fig.2, the transform functions between the physical and the computational regions are chosen as :

$$
\begin{aligned}
\xi & =\frac{x-h_{n}(t)}{h_{n+1}(t)-h_{n}(t)} \\
\eta & =\frac{y-y_{m}(t)}{y_{m+1}(t)-y_{m}(t)} \\
\tau & =t
\end{aligned}
$$

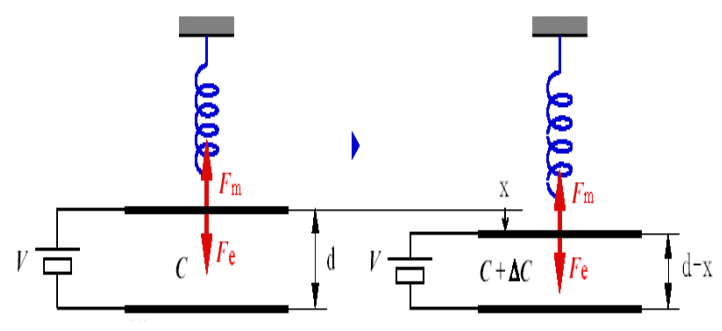

Fig1 Functional model of MEMS capacitor

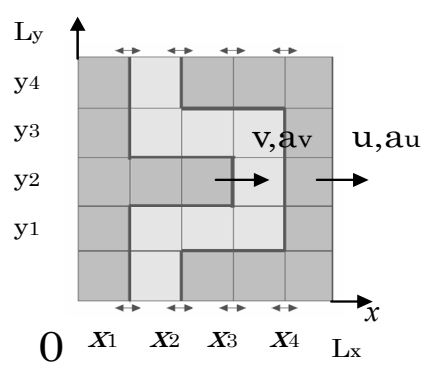

(a)

Fig2 (a)Physical region

(b)Computational region

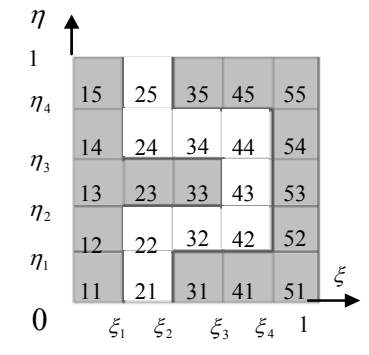

(b) 
,where $\mathrm{n}=1,2,3, \mathrm{~m}=1,2,3$ and $h_{1}(t), h_{2}(t), h_{3}(t), h_{4}(t)$, are written in the following form assuming that the plate accelerations, velocities remain time changing values for the whole time of their motion.

$$
\begin{aligned}
& h_{1}(t)=x_{1}+v t+\frac{1}{2} a_{v} t^{2} \\
& h_{2}(t)=x_{2}+u t+\frac{1}{2} a_{u} t^{2} \\
& h_{3}(t)=x_{3}+v t+\frac{1}{2} a_{v} t^{2} \\
& h_{4}(t)=x_{4}+u t+\frac{1}{2} a_{u} t^{2}
\end{aligned}
$$

The functions $h_{1}(t), h_{2}(t), h_{3}(t), h_{4}(t)$, describe the movement along the $x$ axis, and allow for the realization of a rectangular grid with stationary boundary conditions. The partial time-derivatives in the transformed domain $(\xi, \eta, \tau)$ can be expressed in terms of the partial derivatives of the original domain $(x, y, t)$ using eqs.(4)-(10). The FDTD technique can provide the time-domain solution of the rectangular $(\xi, \eta, \tau)$ grid. The stability criterion in this case is chosen as $c \Delta t \leq \delta / \sqrt{2}$, where $\delta=\Delta x_{0}=\Delta y_{0}$, assuming the grid is uniformly discretized in both directions. In general, $\delta$ is a space increment for $x$ and $y$ direction when the grid increment is minimum (minimum cell size).

\section{Numerical Results}

To validate this approach, numerical results are calculated for a two-dimensional variable capacitor with the movement of the finger only to the $x$-direction. The grid includes $200 \times 200$ cells , input frequency is $\mathrm{f}=20 \mathrm{GHz}$ and $L_{x}=L_{y}=L_{z}=L=5 \lambda, \Delta x=\Delta y=L / 200, \Delta t=3.125 \times 10^{-13}$ (sec). The initial plate separation is $L / 5$ and the grid is terminated with Mur's absorbing boundary conditions. Here, only the left plate is assumed to move due to the coupling of the electrostatic and the mechanical forces. From eq.(3), the acceleration value is derived. Inputting this acceleration value to the transform function $h_{1}(t), h_{3}(t)$, new capacitance $C$ and displacement, transformation function $h_{1}(t), h_{3}(t)$ and acceleration $a_{v}$ are obtained. And then from the capacitance and displacement, new acceleration is obtained. Iterating of this algorithm, it is easy to get the capacitance, acceleration and displacement controlled by the coupling of spring and electrostatic force.

In Fig.3, when the left plate moves away from the right one, the derived mechanical resonant frequencies are shown. Fig.3 displays the time dependence of the acceleration for each mechanical resonant frequency values in the range of $\omega=\sqrt{\frac{k}{m}}=10^{7}(\mathrm{rad} / \mathrm{sec})$ to $\omega=\sqrt{\frac{k}{m}}=10^{\frac{17}{2}}(\mathrm{rad} / \mathrm{sec})$, when the left plate moves away from the right one. The mechanical resonant frequency in Fig 3(a) is $\sqrt{10}$ times of the resonant frequency in Fig3(b) and 10 times of the resonant frequency in Fig3 (c) and also, $10^{\frac{3}{2}}$ times of the resonant frequency in Fig 3(d). According to these values, the ratio of the resonant frequency is shown accurately in each figures. In Fig 3, when the mechanical resonant frequency is low, the amplitude remains almost constant value. Since the typical MEMS device has low mass around $10^{-10} \mathrm{~kg}$ and spring constant around $5-30 \mathrm{~N} / \mathrm{m}$, the mechanical resonant frequency $\omega$ is 
on the order of $10^{5}(\mathrm{rad} / \mathrm{sec})$ [1]. In this paper, the values of the mechanical resonant frequencies are on the order of $10^{7}$, but if the calculation time is taken more, it is possible to get the results around the order of $10^{5}$. The results derived in this paper for acceleration, mechanical resonant frequency are very important values for deciding the switching time.
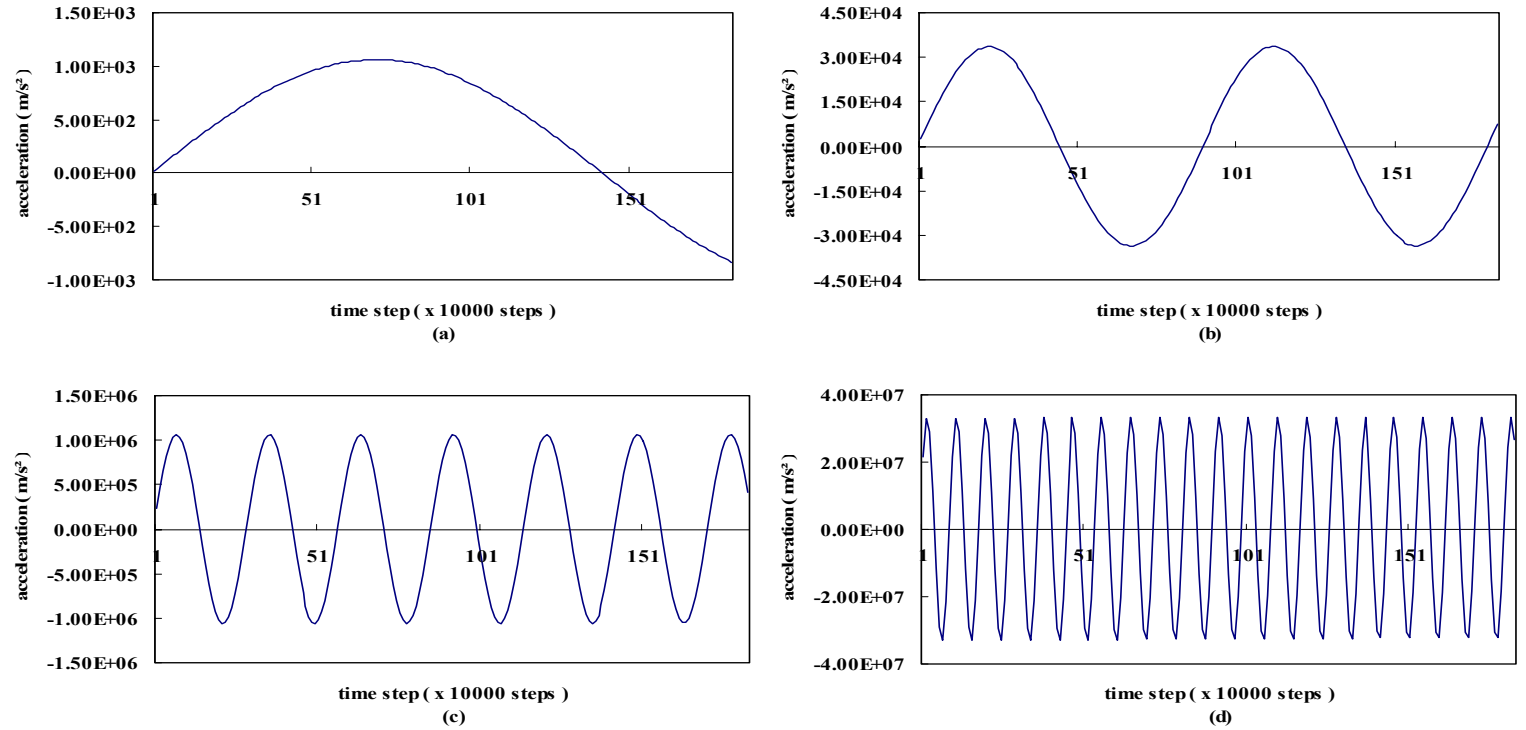

Fig3 Time dependence of the acceleration for each resonant frequency (a) $\omega=10^{7}(\mathrm{rad} / \mathrm{sec}),(b) \omega=10^{\frac{15}{2}}(\mathrm{rad} / \mathrm{sec}),(\mathrm{c}) \omega=10^{8}(\mathrm{rad} / \mathrm{sec}),(\mathrm{d}) \omega=10^{\frac{17}{2}}(\mathrm{rad} / \mathrm{sec}), V=10^{-6}(\mathrm{~V})$

\section{Conclusions}

A novel time-domain modeling technique that has the capability to accurately simulate the transient effect of variable capacitors with accelerated motion controlled by the coupling of the electrostatic and mechanical forces is proposed. Using this technique, the numerical results of the acceleration for each resonant frequency is shown for a MEMS capacitor. The results derived in this paper are very important for deciding the switching time.

\section{Reference}

[1]Gabriel M. Rebeiz, "RF MEMS Theory, Design, and Technology”, John. Wiley \& Sons Publication, 2003.

[2] A. Dec, et-at, "Micromachined Electro-Mechanically Tunable capacitors and Their Applications to RF IC's”, IEEE Trans MTT, pp2587-2596, vol.46, No.12,Dec.,1998

[3]J. F. Thompson, "Numerical grid generation", North Holland, Amsterdam, 1985.

[4] Michiko Kuroda, Noriyuki Miura, Manos M. Tentzeris, "A Novel Numerical Approach for the Analysis of 2D MEMS-Based Variable Capacitors Including the Effect of Arbitrary Motions " ACES Journal, vol.19, no. 1b,pp133-138, March, 2004 